Recepción: 13 / 04 / 2018

Aceptación: 08 / 06 / 2018

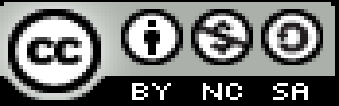

Ciencias técnicas y aplicadas

Publicación: 02 / 07 / 2018

Artículo de investigación

\title{
Diseño conceptual de un sistema de corte industrial mediante el software ANSYS de una máquina cosechadora integral de quinua
}

\section{Conceptual design of an industrial cutting system using the ANSYS software of an integral quinoa harvesting machine}

\section{Projeto conceitual de um sistema de corte industrial utilizando o software ANSYS de uma máquina coletora integral de quinoa}

\author{
Javier E. Orna-Chávez ${ }^{\text {I }}$ \\ javier.orna@espoch.edu.ec \\ Diego F. Mayorga-Pérez II \\ dmayorga@espoch.edu.ec \\ Edwin F. Viteri-Núñez ${ }^{\text {III }}$ \\ eviteri@espoch.edu.ec \\ Otto F. Balseca-Sampedro IV \\ obalseca@espoch.edu.ec \\ Luis I. Sagñay-Quishpe V \\ Israel-luis@live.com \\ Héctor F. Tixe-Guishcaso VI \\ hector.metal@hotmail.es
}

Correspondencia: javier.orna@espoch.edu.ec

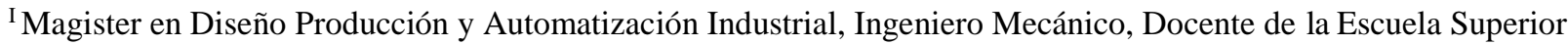
Politécnica de Chimborazo, Riobamba, Ecuador.

II Magister en Seguridad y Prevención de Riesgos del Trabajo, Ingeniero Mecánico, Docente de la Escuela Superior Politécnica de Chimborazo, Riobamba, Ecuador.

III Magister en Gerencia de Proyectos de Ecoturismo, Ingeniero Mecánico, Docente en la Escuela Superior Politécnica de Chimborazo, Riobamba, Ecuador.

IV Magister en sistemas de Transporte de Petróleo y Derivados, Ingeniero Mecánico, Docente en la Escuela Superior Politécnica de Chimborazo, Riobamba, Ecuador.

v Ingeniero Mecánico, Egresado de la Escuela Superior Politécnica de Chimborazo, Riobamba, Ecuador.

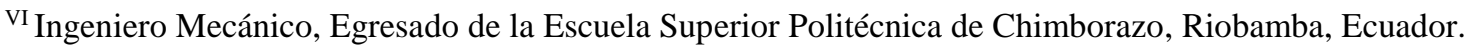




\section{Resumen}

La quinua es un cultivo que se ha incrementado en Ecuador; las provincias con mayor producción son Carchi con $33 \%$, Imbabura con $27 \%$ y Chimborazo con $15 \%$, por lo cual es de necesidad regional que haya máquinas para su cosecha, reduciendo así tiempos y costos de mano de obra. El desarrollo del proyecto tuvo como objetivo diseñar el estudio de un sistema de corte para una mini máquina cosechadora de quinua de $2 \mathrm{~kg} / \mathrm{s}$ de mies sin trillar (panojas), la cual servirá como aporte para futuras investigaciones y una futura construcción. Para ello, se usó una metodología de tipo explicativa y de campo, dado que su interés se centró en el diseño de la primera parte de las cinco que conforma la mini máquina cosechadora de quinua, como es el sistema de corte, siendo el encargado de cortar y recolectar. La información se obtuvo a través de la técnica de la entrevista a los agricultores y técnicos de las procesadoras de quinua. Los resultados del trabajo, permitieron dimensionar las piezas que conforman el sistema de corte y reforzar el cálculo del análisis de resistencia en el software ANSYS. Entre sus conclusiones, se presenta un diseñó del modelo del sistema de corte o cabezal, siendo la primera parte de la máquina cosechador integral de quinua.

Palabras clave: sistema de corte; quinua; software ANSYS; capacidad del sistema; corte por impacto.

\section{Abstract}

Quinoa is a crop that has grown in Ecuador, the provinces with the highest production are Carchi with 33\%, 27\% Imbabura and Chimborazo with 15\%, which is of regional need machines to their harvest, thus reducing time and labor costs. The project aimed to design the study of cutting for a mini machine system combine quinoa from $2 \mathrm{~kg} / \mathrm{s}$ of harvest without thresh (panicles), which will serve as input for further research and a future construction. To do this, a methodology of explanatory type and field, was used since their interest focused on the design of the first part of the five that conforms the mini machine combine quinoa, as it is cut, being responsible for cutting and collecting system. The information was obtained through the technique of the interview to the farmers and the processors of quinoa technicians. The results of work, allowed size parts that make up the cutting system and reinforce the calculation of resistance in ANSYS software analysis. Among their conclusions, presented a designed system of cutting or head model, being the first part of the machine Harvester integral of quinoa. 
Keywords: cutting; quinoa; ANSYS software; system capacity; impact cutting system.

\section{Resumo}

Quinoa é uma cultura que cresceu no Equador, as províncias com a maior produção são Carchi com $33 \%$, Imbabura com $27 \%$ e Chimborazo com 15\%, o que é uma necessidade regional para ter máquinas de colheita, vezes, reduzindo assim e custos trabalhistas. O projecto foi para a concepção do estudo de um sistema de corte para uma máquina de colheita de mini quinoa $2 \mathrm{~kg} /$ s de colheita sem limiar (panículas), que vai servir como entrada para futura pesquisa e construção futuro. Portanto, uma metodologia explicativo e tipo de campo é utilizado, uma vez que o foco foi sobre o desenho da primeira parte do cinco formar o mini-quinoa debulhadora, como é o sistema de corte ser responsável pela cortar e recolher. A informação foi obtida através da técnica de entrevistar os agricultores e técnicos dos processadores de quinoa. Os resultados do trabalho permitiram dimensionar as peças que compõem o sistema de corte e reforçar o cálculo da análise de resistência no software ANSYS. Entre suas conclusões, apresenta-se um desenho do modelo do sistema de corte ou cabeçote, sendo a primeira parte de toda a máquina de colheita de quinoa.

Palavras chave: sistema de corte; quinoa; software ANSYS; capacidade do sistema; corte de impacto.

\section{Introducción}

En la actualidad el aumento de la demanda de quinua crece continuamente, por lo que el diseño de una máquina cosechadora integral incrementaría la producción en un valor considerable. La máquina cosechadora posee cinco partes constitutivas: sistema de corte, trillado, eléctrico y electrónico, hidráulico, y transmisión. La cosecha de las panojas de quinua se ha venido realizando por medio de una cosecha manual con una hoz para el corte de la planta, conllevando gran tiempo y esfuerzo humano siendo un problema para el aumento de la producción, afectando de esta manera a la calidad de vida de los pequeños agricultores por el precio bajo que reciben de la venta del producto. Dentro de las características del cultivo de quinua se pueden distinguir las del tallo, de las panojas, distancia entre siembra y el rendimiento del cultivo.

Características del tallo. Es cilíndrico en el cuello de la planta y un ángulo a partir de las ramas, puesto que las hojas son alternas dando una configuración excepcional. El grosor del tallo 
también es variable. Dependiendo del genotipo, siembra y disponibilidad de nutrientes, expresa la INIA (2013), que la coloración del tallo es variable, desde el verde al rojo, presenta estrías y también axilas pigmentadas de color rojo, o púrpura. El tallo contiene gran cantidad de pectina y celulosa que se puede utilizar en la fabricación de papel y cartón.

Características de las panojas. Es una panoja típica, constituida por un eje central y ramificaciones secundarias, terciarias y pedicelos que sostienen a los glomérulos. El eje principal está más desarrollado que los secundarios, ésta puede ser laxa (Amarantiforme) o compacta (glomerulada), existiendo formas intermedias entre ambas. (Cervilla, Mufari y Guzmán, 2011).

Distancia entre siembra. La variedad que más siembra son de dos tipos, expresan (Peralta, Mazón, Murillo y Rivera, 2012), por ser variedades mejoradas las cuales son las siguientes: INIAP TUNKAHUAN (dulce, baja en saponina), INIAP Pata de Venado o Taruka Chaki (dulce, baja en saponina), estos tipos de semillas mejoradas, tienen un cicló de cultivo, INIAP Tunkahuan de 150 a 170 días; INIAP Pata de Venado de 130 a 150 días, la cantidad de semilla apropiada por hectárea esta entre 12 a 16kg, la distancia entre surcos es de $60 \mathrm{~cm}$ para Tunkahuan y $40 \mathrm{~cm}$ para Pata de Venado, siempre que se lo realice manualmente.

Rendimiento del cultivo. En el Ecuador las provincias con mayor producción de quinua son las siguientes: Carchi, Chimborazo, Imbabura y Pichincha, los cuales de acuerdo a las estadísticas del MAGAD siembran alrededor de dos mil hectáreas al año con una producción total de 1400 toneladas con un promedio de 1,27 ton/ ha, en la provincia de Chimborazo. Guerrero (2016).

Tabla 1. Parámetros del cultivo de la planta.

\begin{tabular}{ll}
\hline Diámetro del tallo & De 2,10 a $2,5 \mathrm{~cm}$ \\
\hline $\begin{array}{l}\text { Longitud de la } \\
\text { panoja }\end{array}$ & $33,80 \mathrm{~cm}$ \\
Diámetro de la & $8,60 \mathrm{~cm}$ \\
panoja & \\
Longitud de & $7,70 \mathrm{~cm}$ \\
glomérulos & \\
Número de panojas & 1 \\
por planta
\end{tabular}




\begin{tabular}{ll}
\hline $\begin{array}{l}\text { Distancia entre } \\
\text { siembra }\end{array}$ & $\begin{array}{l}\text { De } 40 \text { a } 60 \mathrm{~cm} \\
\text { entre hileras }\end{array}$ \\
$\begin{array}{l}\text { Rendimiento por } \\
\text { hectárea en la } \\
\text { provincia. }\end{array}$ & \\
\hline
\end{tabular}

Fuente: Elaboración propia

Con base a lo expuesto, se presenta un sistema de corte de una máquina cosechadora integral de quinua, para una producción de corte de $2 \mathrm{~kg} / \mathrm{s}$ de mies sin trillar (panojas), para ello se utilizaron las ecuaciones de los libros de diseño mecánico y de máquinas agrícolas.

\section{Metodología}

La investigación siguió una metodología de tipo explicativa y de campo, dado que su interés se centró en el diseño de la primera parte de las cinco que conforma la mini máquina cosechadora de quinua, como es el sistema de corte, siendo el encargado de cortar y recolectar. La información se obtuvo a través de la técnica de la entrevista a los agricultores y técnicos de las procesadoras de quinua, recopilando los datos necesarios para el dimensionamiento y análisis de resistencia de los elementos que conformaran el sistema de corte. Asimismo, el diseño del sistema de corte de la máquina, considero los siguientes postulados, tal como se especifica a continuación:

Identificación de la necesidad. La primera fase, presenta la necesidad del cliente o del jefe, el enunciado será breve y sin detalle, es decir, "lo que se necesita es". No debe constar un planteamiento estructurado del problema.

Investigación previa. Esta fase se la considera la más importante, pues, es una etapa donde se investiga de manera mundana, los aspectos pertinentes del problema. Además, es pertinente indagar si éste o un problema similar ya han sido resueltos con anterioridad. Por lo que la revisión de patentes, plantea Norton (2009), es un punto importante de considerar en el proceso de diseño.

Diseño conceptual. Es la etapa donde se analizan los diferentes conceptos de funcionamiento, opciones de diseño, entre otras, es decir: identificación de los problemas esenciales a tratar, 
establecer las especificaciones, la estructura funcional, el diseño modular, el aseguramiento de la calidad, y por último la etapa de criterios de selección.

Diseño preliminar. Esta etapa es de determinación y concretización de las componentes en su forma específica, materiales propuestos, el tiempo de funcionamiento, las velocidades de los diferentes sistemas, las dimensiones de los elementos que conforma la máquina. En otras palabras, las especificaciones del diseño, es decir, como se debe hacer.

Diseño detalle. Esta fase es de detalle y especificación, en la elaboración de planos, la determinación de etapa de fabricación, los proveedores, establecimiento de los costos de fabricación y construcción. Es la etapa más desarrollado a nivel empresarial.

Documentación. Esta etapa se recopila toda la información procesada, de forma ordenada, para la presentación final. Aplicación de la norma INEN "Documentación, presentación de tesis, trabajos de grado y otros trabajos de investigación”. Hamrock, Jacobson y Schmid (2000).

\section{Resultados}

\section{En relación al diseño del sistema de corte}

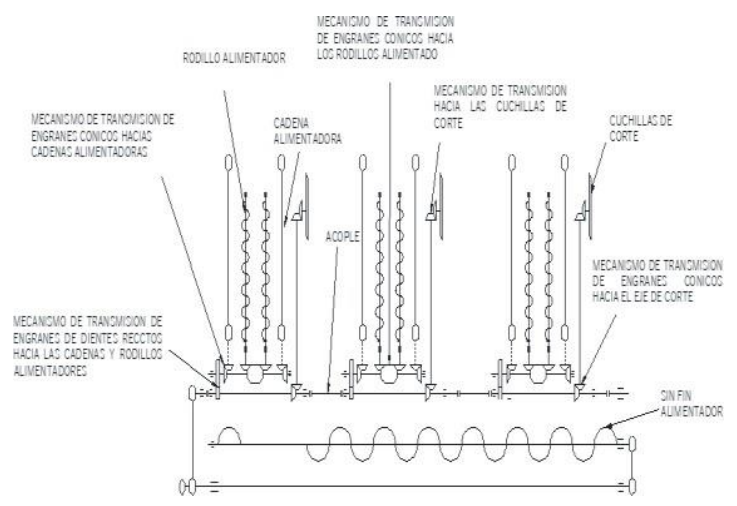

Figura 1. Esquema cinemático del sistema de corte.

\section{Capacidad del sistema}

Se determina de la siguiente ecuación donde $\mathrm{v}_{\mathrm{m}}$ es la velocidad de avance de la máquina es igual a $4 \mathrm{~km} / \mathrm{h}$ según lo recomendado, y B es el ancho de corte de trabajo con valor igual a $2 \mathrm{~m}$. 


$$
\mathrm{C}_{\mathrm{t}}=0,1 * \mathrm{v}_{\mathrm{m}} * \mathrm{~B}
$$

$$
\mathrm{C}_{\mathrm{t}}=0,80 \mathrm{ha} / \mathrm{h}
$$

\section{Producción teórica de corte.}

$$
\mathrm{C}_{\mathrm{p}}=\mathrm{P}_{\mathrm{ha}} * \mathrm{C}_{\mathrm{t}}
$$

\section{Tiempo de trabajo operativo.}

$$
\mathrm{t}_{\mathrm{op}}=\frac{1}{\mathrm{n} * \mathrm{Ct}}
$$

Para una eficiencia del $n=80 \%$ de acuerdo a recomendaciones propuestas por Ortiz (2012).

\section{Diseño de cuchillas de corte}

\section{Cálculo de la velocidad angular de corte.}

$$
\mathrm{w}>\frac{\mathrm{v}_{\mathrm{k}}+\mathrm{v}_{\mathrm{m}}}{2 \mathrm{r}}
$$

Donde $\mathrm{v}_{\mathrm{m}}$ es la velocidad de desplazamiento de la máquina e igual a 1,1 m/s, según Ortiz (2012). $\mathrm{V}_{\mathrm{k}}$ es la velocidad para realizar un corte limpio la cual se tomó como referencia a las velocidades del corte de caña y un valor medio del mínimo igual a $10 \mathrm{~m} / \mathrm{s}, \mathrm{r}$ es el radio de la cuchilla igual a $60 \mathrm{~mm}$.

$\mathrm{w}>884,19 \mathrm{rpm}$

De donde se toma que la velocidad será de w=900 rpm o n=900rpm.

\section{Cálculo del número de cuchillas.}

$$
z=\frac{2 V_{m}}{h w}
$$

Donde h es la altura de la parte cortante del segmento e igual a $130 \mathrm{~mm}$.

$$
\mathrm{z}=0,1811
$$

Pero para el diseño se escogió dos segmentos $\mathrm{z}=2$. 


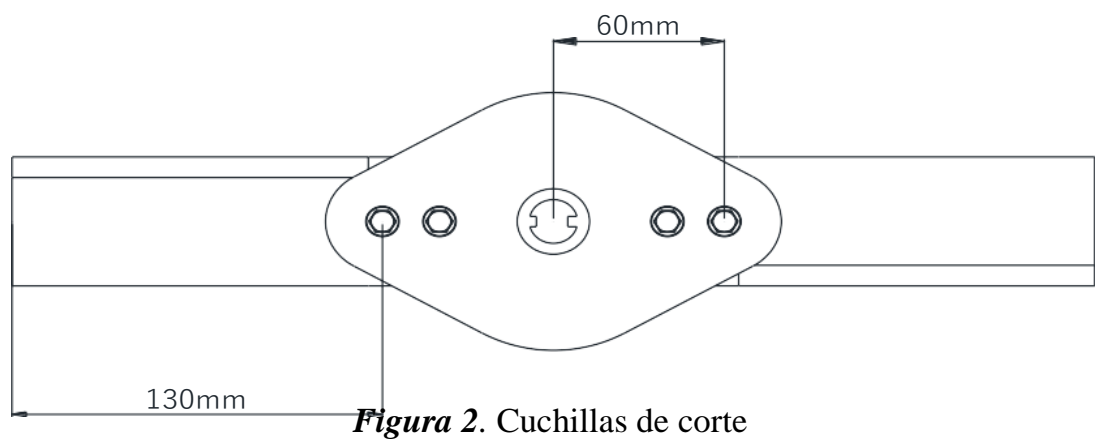

\section{Cálculo de la fuerza de corte}

El esfuerzo de corte, para LLangari y Benalcazar (2012) es de $1 \mathrm{kgf} / \mathrm{cm}^{2}$ y el area es de $\mathrm{A}_{\mathrm{q}}=1,27$ $\mathrm{cm}^{2}$ aproximadamente.

$\mathrm{F}=F_{\mathrm{c}} * A$

Para lo cual se tiene que el area de corte será la de aproximadamente 10 plantas de donde $\mathrm{A}=$ $12,7 \mathrm{~cm}^{2}$ obteniendo lo siguiente:

$$
\mathrm{F}=12,67 \mathrm{kgf}(124,283 \mathrm{~N})
$$

\section{Cálculo del torque necesario para el corte}

$\mathrm{T}=\mathrm{F} * \mathrm{r}_{1}$

Donde $\mathrm{r}_{1}$ es el radio promedio de corte e igual a $0,15 \mathrm{~m}$

$$
T_{a c}=1,9 \mathrm{kgf}-\mathrm{m}(18,64 \mathrm{~N}-\mathrm{m})
$$

\section{Cálculo del espesor de la cuchilla}

El límite de fluencia a corte puro, expuesto por Budynas y Nisbett (2012).

$$
\mathrm{S}_{\mathrm{sy}}=0,577 \mathrm{~S}_{\mathrm{y}}
$$

El material utilizado para la cuchilla es un acero AISI 5160 con un limite de fluencia de 510 Mpa.

De donde : 
$\tau_{\max }=\frac{\mathrm{S}_{\mathrm{sy}}}{\mathrm{n}}$

$$
\begin{gathered}
\tau_{\max }=\frac{\mathrm{F}_{\mathrm{c}}}{\mathrm{h} * \mathrm{e}} \\
\mathrm{e}=0,0813 \mathrm{~mm}
\end{gathered}
$$

Cálculo de la potencia necesaria para la cuchilla de corte.

$$
\begin{aligned}
& \mathrm{P}=\frac{\mathrm{T}_{\mathrm{ac}} * \mathrm{n}}{7160} \\
& \mathrm{P}_{\text {corte }}=2,36 \mathrm{hp}
\end{aligned}
$$

\section{Determinación de la potencia de cadenas alimentadoras}

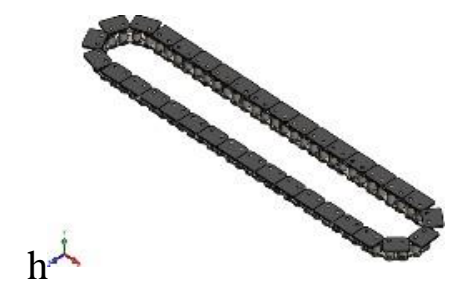

Figura 3. Cadenas alimentadoras de mies

\section{Determinación de la velocidad angular de la cadena alimentadora.}

La velocidad de la cadena es aproximadamente 25 a 50 \% más rápido que la velocidad de avance de la máquina cuando los tallos están en posición vertical. [10]

$v_{m}=w_{c a d} \cdot r_{c a d}$

$w_{\text {cad }}=15,86 \mathrm{rad} / \mathrm{seg}=151,58 \mathrm{rpm}$

Para el diseño se aplicó una velocidad de cadenas alimentadoras de $200 \mathrm{rpm}$, y por lo tanto una velocidad de la cadena de 1,47 m/seg, siendo de este modo $36 \%$ más rapido que el avance la máquina

Determinación de la potencia para la cadena alimentadora.

$$
P_{\text {cad-alim }}=\mathrm{Q} * \mathrm{H}+\mathrm{Q} * \mathrm{f} * \mathrm{~L}_{\text {carga }}+\mathrm{q}_{1} * \mathrm{f} * \mathrm{~L}_{\text {total }} \quad \text { (12) }
$$

Ya que la cadena transporta carga la ecuación (12) se resume en: 
$P_{\text {cad-alim }}=q_{1} * f * L_{\text {total }}$

$\mathrm{q}_{1}=0,85 \mathrm{Kgf} / \mathrm{m}$ se determinó mediante el software Solidworks, $\mathrm{f}=0,33, \mathrm{~L}_{\text {total }}=0,7 \mathrm{~m}$. Con lo cual se determinó la fuerza de tracción:

$P_{\text {cad-alim }}=0,19635 \mathrm{kgf}$

Una vez determinado la fuerza de tracción, se calcula la potencia de la cadena alimentadora mediante la ecuación (13).

$$
N_{t}=\frac{P * v}{75}
$$

Donde P es la fuerza de tracción y v la velocidad de la cadena.

$N_{t}=0,00385 \mathrm{hp}$

Pero se consideró para el diseño una potencia de $0,01 \mathrm{hp}$.

\section{Diseño de rodillos transportadores}

Las longitudes de los rodillos oscilan generalmente entre 1 y 1,25m y sus diámetros son de 7,5 a $10 \mathrm{~cm}$, las velocidades periféricas suelen ser de 180m/min y una separación de rodillo de 6 a $13 \mathrm{~mm}$, pero seran modificados de acuerdo a las condiciones de trabajo. [10]

$v_{\text {rod }}=w_{\text {rod }}, r_{\text {rod }}$

Para el diseño se aplicó una velocidad de rodillo alimentador de 800 rpm.

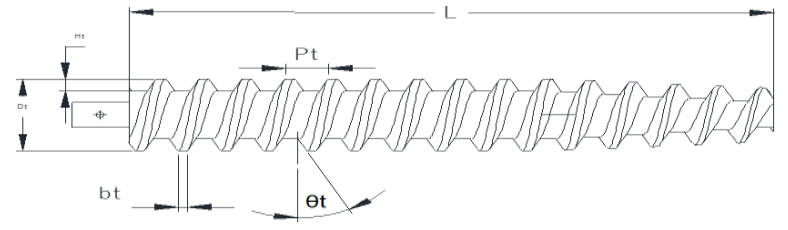

Figura 4. Esquema de rodillos transportadores de mies

\section{Dimensionamiento del rodillo alimentador}

\section{Cálculo del diámetro del rodillo}


$D_{t}^{3}=\frac{0,0265256 * C_{p}}{\omega_{t} C_{f} \delta C_{i}}$

Donde $\omega_{\mathrm{t}}: 800 \mathrm{rpm}$ velocidad del rodillo alimentador, $\mathrm{C}_{\mathrm{f}}: 0.45, \delta=0.49 \mathrm{gr} / \mathrm{cm}^{3}$ y $\mathrm{C}_{\mathrm{i}}=1$ al no estar inclinado

$\mathrm{C}_{\mathrm{p}}=1,2$ ton/hora

$D_{t}=0,072 m$

Por condiciones geometricas se tomó un $\mathrm{D}_{\mathrm{t}}=0,072 \mathrm{~m}$

\section{Cálculo del diá metro en tubo del eje del transportador}

$E_{s}=K * D$

El factor se toma $\mathrm{K}$ es igual a 0,44 . [11]

$E_{s}=0,032 m$

\section{Cálculo del paso $P_{t}$ del rodillo alimentador}

$P_{t}=F_{p} * D_{t}$

El factor $F_{p}$ es igual a 0,8 para paso normal:

$P_{t}=0,0576 \mathrm{~m}$

Para el diseño se optó un paso de $0.03 \mathrm{~m}$ porque el objetivo del rodillo alimentador es de dar una ligera presión a las plantas para su posterior corte.

\section{Cálculo del espesor del cuerpo del transportador}

$E c=0,0104166 * D_{t}$

$E c=b_{t}=0,75 \mathrm{~mm}$

\section{Cálculo de la potencia para el rodillo alimentador}

La potencia necesaria se determina sumando las distintas potencias, las cuales son: por fricción del transporte, por el material transportado y por la inclinación del transporte. Las ecuaciones estan basadas en el catalogo de Martin Material Handling. 
La ecuación que se aplica para la determinación de la potencia por la fricción es la siguiente:

$P_{f}=\frac{L * \omega * F_{d} * F_{b}}{1000000}$

Donde:

$\mathrm{L}: 2,30$ pies [ft], $\omega: 800$ [rpm], Fb: 1, Fd: 12,

Reemplazando los valores en la ecuación (18) se obtiene el siguiente valor:

$P_{f}=0,022 h p$

Determinación de la potencia por el material a transportar:

Donde:

$P_{m}=\frac{c * L * \delta * F_{f} * F_{m} * F_{p}}{1000000}$

c: $172,09\left[\mathrm{ft}^{3} /\right.$ hora $], \mathrm{L}: 2,3[\mathrm{ft}], \delta: 30,75\left[\mathrm{lbs} / \mathrm{pie}^{3}\right], \mathrm{F}_{\mathrm{f}}: 1, \mathrm{~F}_{\mathrm{m}}: 2, \mathrm{~F}_{\mathrm{p}}: 1$,

Reemplazando en la ecuacion (19) tenemos la potencia por el material a transportar:

$\mathrm{P}_{\mathrm{m}}=0,012 \mathrm{hp}$

Al no tener inclinación la potencia por la inclinación del transporte es nula.

Entonces la potencia necesaria se determina por la siguiente ecuacion:

$P_{\text {trans }}=\frac{\left(P_{m}+P_{f}+P_{i}\right) F_{o}}{e}$

Donde:

$\mathrm{F}_{\mathrm{o}}$ : factor de sobrecarga 2,75 , e: eficiencia $80 \%$.

$\mathrm{P}_{\text {trans }}=0,106 \mathrm{hp}$

Potencia debido a la inercia del rodillo alimentador:

$P_{\text {inercia }}=\frac{\left(T_{a c}\right) * n}{7160}$ 
$\mathrm{T}_{\mathrm{ac}}$ : Torsión generado por aceleración [N.m]

$\mathrm{n}$ : revoluciones por minuto; $\mathrm{n}=800 \mathrm{rpm}$

$T_{a c}=I * \alpha$

I: $0,003879 \mathrm{Kg} \cdot \mathrm{m}^{2} ; \alpha=2,11 \mathrm{rad} / \mathrm{seg}^{2}$

$$
\begin{aligned}
& \mathrm{T}_{\mathrm{ac}}=0,00215 \mathrm{~N} \cdot \mathrm{m} \\
& \mathrm{T}_{\mathrm{ac}}=0,00215 \mathrm{~N} \cdot \mathrm{m}
\end{aligned}
$$

De acuerdo a la ecuacion (10) se llega a determinar la potencia debido a la inercia del rodillo.

$P_{\text {inercia }}=0,00239 \mathrm{hp}$

La potencia total del rodillo sera igual a la potencia necesaria para moverla en vacio sumada la potencia para realizar el trabajo.

$$
\begin{gathered}
P_{\text {rod }}=P_{\text {inercia }}+P_{\text {trans }} \\
P_{\text {rod }}=0,106 \mathrm{hp}
\end{gathered}
$$

\section{Diseño de caja de engranes}

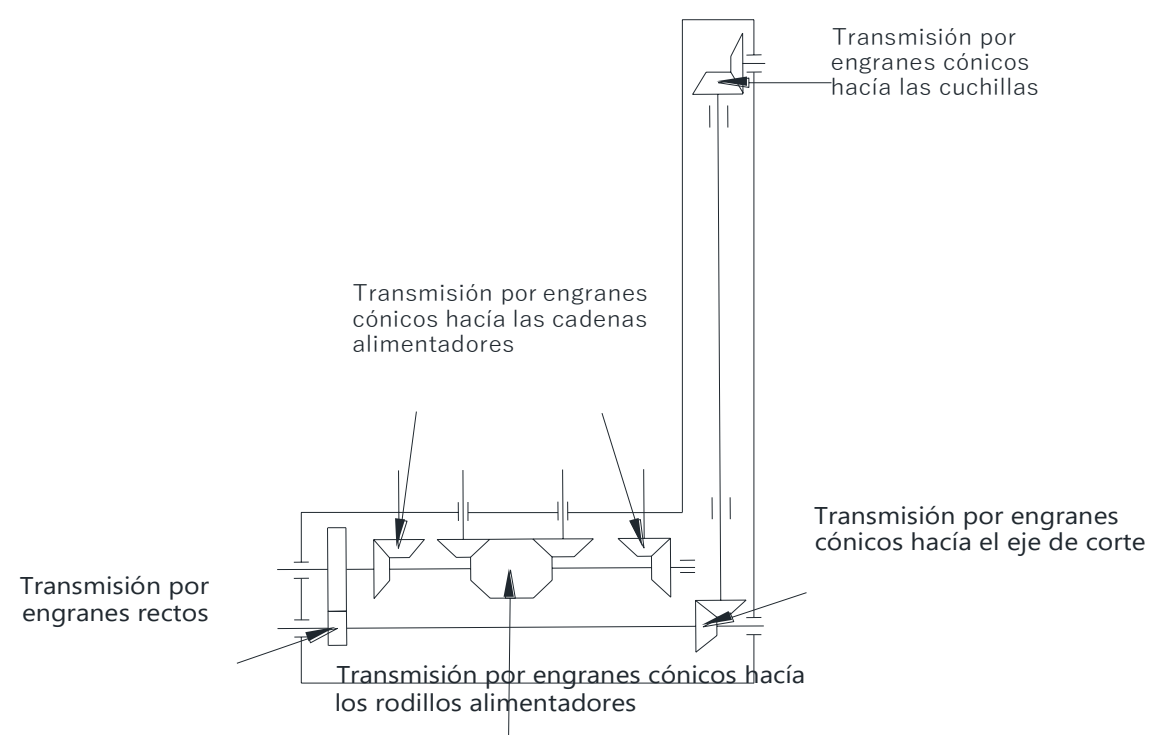

Figura 5. Esquema de la caja de engranajes. 


\section{Diseño de engranes rectos}

Determinación del torque

Las potencias que se transmiten a los engrane es la suma de las potencias a suministrar a las cadenas y a los rodillos alimentadores las:

$\mathrm{P}_{\text {trans }}=0,232 \mathrm{hp}$

La velocidad angular del eje donde se encuentran acoplados los engranes en condiciones de trabajo es de $600 \mathrm{rpm}$.

Aplicando estos datos en la ecuación (10) se determinó el torque al cual estará sometido el par de engranes:

$\mathrm{T}_{\mathrm{acp}}=2,77 \mathrm{~N} . \mathrm{m}$

\section{Cálculo de la fuerza tangencial y radial en los dientes.}

La fuerza tangencial se lo determina aplicando la siguiente ecuación.

$$
W^{t}=33000 \frac{H}{V_{t}}
$$

$$
W_{t}=27,54 l b f
$$

Aplicando la ecuación (22) Se determina la fuerza en dirección radial.

$W_{r}=W_{t} \tan \varphi$

Donde $\phi$ es el ángulo de presión e igual $20^{\circ}$ sustituyendo se encuentra el valor de la fuerza radial.

$$
W_{r}=10,02 l b f
$$

\section{Esfuerzo por flexión}

Se aplica las ecuaciones de la norma AGMA 2001:D04 para determinar la resistencia en el engrane. [12] 
$\sigma=W^{t} K_{o} K_{v} K_{s} \frac{P_{d}}{F} \frac{K_{m} K_{B}}{J}$

$$
\sigma_{p}=1177,52 \mathrm{psi}
$$

\section{Esfuerzo permisible de flexión}

El esfuerzo permisible de flexión no es más que la resistencia del material del engrane, ya que el objetivo del cálculo es la determinación del factor de seguridad.

$\sigma_{\text {perm }}=\frac{S_{t}}{S_{F}} \frac{Y_{N}}{K_{T} K_{R}}$

Número de esfuerzos de flexión permisible del acero AISI 1015 de grado 1 cementado, de duerza $\mathrm{HBP}=250 \mathrm{y} \mathrm{HBG}=250$.

$S_{t}=77,3 H_{B}+12800 p s i$

$$
\left(\sigma_{f p}\right)_{a d m}=30293,875 p s i
$$

\section{Cálculo del factor de seguridad a flexion.}

El factor de seguridad a flexión es la división del esfuerzo de flexión permisible entre el esfuerzo de flexión como se indica en la siguiente ecuación:

$$
S_{F p}=\frac{S_{t} Y_{N} /\left(K_{T} K_{R}\right)}{\sigma}
$$

$$
S_{\mathrm{Fp}}=25,73
$$

\section{Esfuerzo por contacto}

Tambien conocido como falla por fatiga superficial en los dientes, debido a las elevadas cargas de contacto superficial que remueven las particulas en la superficie.

$$
\sigma_{c}=C_{p} \sqrt{W^{t} K_{o} K_{v} K_{s} \frac{K_{m}}{d_{p} F} \frac{C_{f}}{I}}
$$

$$
\sigma_{\mathrm{cp}}=32929,61 \mathrm{psi}
$$




\section{Esfuerzo permisible de contacto}

$$
\sigma_{\text {cperm }}=\frac{S_{c}}{S_{H}} \frac{Z_{N} C_{H}}{K_{T} K_{R}}
$$

Número de esfuerzos de flexión permisible del acero AISI 1015 de grado 1 cementado, de duerza $\mathrm{HBP}=250 \mathrm{y} \mathrm{HBG}=250$.

$$
\mathrm{S}_{\mathrm{c}}=322 \mathrm{H}_{\mathrm{B}}+29100 \mathrm{psi}
$$

$$
\left(\sigma_{c p}\right)_{a d m}=102037.6 p s i
$$

De donde se obtiene el factor de seguridad por contacto.

$$
S_{H p}=\frac{S_{c} Z_{N} / K_{T} K_{R}}{\sigma_{c}}
$$

$$
S_{H p}=3,1
$$

\section{Diseño de engranes cónicos}

Se aplica las ecuaciones de la norma AGMA 2003-B97 para determinar la resistencia en los engranes. [12]

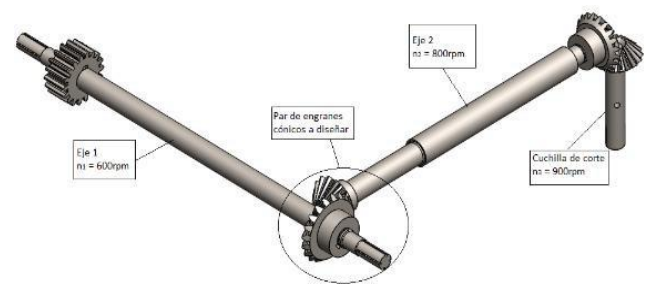

Figura 6. Esquema de la trasmisión por engranes cónicos.

La potencia a transmitir es la potencia necesaria para el corte limpio de la planta de quinua, lo cual fue calculado anteriormente en el inciso anterior al igual que la velocidad angular.

$\mathrm{P}_{\text {corte }}=2,36 \mathrm{hp} \quad \mathrm{n}_{1}=600 \mathrm{rpm}$

\section{Torque generado}

Despejando y aplicando los valores calculados en la ecuación (10) se tiene: 


$$
\mathrm{T}_{\mathrm{p}}=28,11 \mathrm{~N} . \mathrm{m}
$$

Fuerza tangencial $\left[\mathbf{W}_{t}\right]$

$$
W^{t}=33000 \frac{h p}{v_{t}}
$$

$$
W^{t}=198,32 l b f
$$

\section{Fuerza radial $\left[\mathrm{W}_{\mathbf{r}}\right]$}

$W_{r}=W_{t} \cos \gamma \tan \varphi$

$\phi$ : Angulo de presión; $\phi=20^{\circ} \mathrm{C}$

$$
W_{r}=43,31 \mathrm{lbf}
$$

\section{Fuerza axial $\left[\mathbf{W}_{\mathrm{a}}\right]$}

$$
W_{\mathrm{a}}=\mathrm{W}_{\mathrm{t}} \sin \gamma \tan \emptyset
$$

$$
\mathrm{W}_{\mathrm{a}}=57,75 \mathrm{lbf}
$$

\section{Esfuerzo en engranes cónicos}

Esfuerzo de flexión

$$
S_{t}=\frac{W^{t}}{F} P_{d} K_{o} K_{v} \frac{K_{g} K_{m}}{K_{x} J}
$$

$$
S_{t-p}=6265,005 p s i
$$

Esfuerzo permisible de flexión

$$
S_{w t}=\frac{S_{a t} K_{L}}{S_{F} K_{T} K_{R}}
$$

Para el cálculo del número de esfuerzo permisible de la AGMA se utilizo el acero AISI 1045, templado y revenido, con una dureza de $610 \mathrm{HBr}$ para grado 2.

$S_{a t}=48 H_{B}+5980$

$S_{w t-p}=33251,97 \mathrm{psi}$ 
Factor de seguridad a flexión

$$
S_{F p}=5,27
$$

\section{Cálculo de esfuerzo a contacto}

$$
S_{c}=\sigma_{c}=C_{p} \sqrt{\frac{W^{t}}{F d_{p} I} K_{o} K_{v} K_{m} C_{s} C_{x c}}
$$

$$
\sigma_{\mathrm{c}}=95710.44 \mathrm{psi}
$$

\section{Esfuerzo permisible de contacto}

$$
S_{w c}=\left(\sigma_{c}\right)_{\text {perm }}=\frac{S_{a c} C_{\mathrm{L}} C_{\mathrm{H}}}{S_{\mathrm{H}} K_{\mathrm{T}} K_{\mathrm{R}}}
$$

Número de esfuerzo permisible de la AGMA se utilizo el acero AISI 1045, templado y revenido, con una dureza de $610 \mathrm{HBr}$ grado 2 .

$$
S_{a c}=363.6 H_{B}+29560
$$

$$
S_{w c-p}=256407,99 p s i
$$

Determinación del factor de seguridad

$$
S_{H p}=2.67
$$

Los cálculos realizados se presentan con más detalle en las tablas siguientes:

Tabla 2. Resultados del diseño engranes conicos para eje de corte

\begin{tabular}{|l|c|c|}
\hline Potencia & 2,36 & $\mathrm{Hp}$ \\
\hline Torque $\mathrm{T}_{\mathrm{p}}$ & 249,22 & $\mathrm{lbf}-\mathrm{in}$ \\
\hline \multicolumn{3}{|c|}{ Diseño a flexión } \\
\hline & Piñón & Rueda \\
\hline Esfuerzo por Flexión & $6,32 \mathrm{ksi}$ & $7,26 \mathrm{ksi}$ \\
\hline \multicolumn{3}{|c|}{ Esfuerzo permisible Flexión } \\
\hline \multirow{2}{*}{ Tipo de acero } & \multicolumn{2}{|c|}{$61045 \mathrm{AISI}$} \\
\cline { 2 - 3 } & $\mathrm{HBN}$ \\
\cline { 2 - 3 } & $33.25 \mathrm{ksi}$ & $33,08 \mathrm{ksi}$ \\
\hline Esfuerzo flexión & 5,26 & 4,56 \\
\hline Factor de seguridad a
\end{tabular}




\begin{tabular}{|l|c|c|}
\hline \multicolumn{3}{|c|}{ Esfuerzo a contacto } \\
\hline Esfuerzo a contacto & $96,13 \mathrm{ksi}$ & $96,13 \mathrm{ksi}$ \\
\hline \multicolumn{3}{|c|}{ Esfuerzo permisible de contacto } \\
\hline Esfuerzo permisible & $256,42 \mathrm{ksi}$ & $252 \mathrm{ksi}$ \\
\hline Factor de seguridad & 2,67 & 2,62 \\
\hline
\end{tabular}

Fuente: Elaboración propia

Tabla 3. Resultados del diseño de engranes cónicos (cuchillas de corte)

\begin{tabular}{|l|c|c|}
\hline Potencia & 2,36 & Hp \\
\hline Torque $\mathrm{T}_{\mathrm{p}}$ & 186,91 & lbf-in \\
\hline \multicolumn{2}{|c|}{ Diseño a flexión } \\
\hline \multicolumn{2}{|c|}{ Esfuerzo permisible Flexión } \\
\hline Esfuerzo por Flexión & $6,05 \mathrm{psi}$ & Rueda \\
\hline \multicolumn{2}{|c|}{1045 AISI } \\
\hline \multirow{2}{*}{ Tipo de acero } & 610 & HBN \\
\cline { 2 - 3 } & $33,08 \mathrm{psi}$ & $33,01 \mathrm{psi}$ \\
\hline Esfuerzo a flexión & 5,47 & 5,86 \\
\hline Factor de seguridad a & Esfuerzo a contacto \\
\hline \multicolumn{3}{|c|}{ Esfuerzo permisible de contacto } \\
\hline Esfuerzo a contacto & $25,6 \mathrm{ksi}$ & $86,6 \mathrm{ksi}$ \\
\hline \multicolumn{2}{|c|}{ Esfolksi } & $250,23 \mathrm{ksi}$ \\
\hline Esfuerzo permisible & 2,91 & 2,89 \\
\hline Factor de seguridad
\end{tabular}

Fuente: Elaboración propia

Tabla 4. Resultados del diseño los engranes cónicos (cadenas alimentadoras)

\begin{tabular}{|c|c|c|}
\hline \begin{tabular}{l|l} 
Torque Tp & 2,11 lbf-
\end{tabular} & Potencia & $0,01 \mathrm{HP}$ \\
\hline \multicolumn{3}{|c|}{ Diseño a flexión } \\
\hline & Piñón & Rueda \\
\hline Esfuerzo por Flexión & $0,124 \mathrm{ksi}$ & $0,146 \mathrm{ksi}$ \\
\hline \multirow{2}{*}{ Tipo de acero } & \multicolumn{2}{|c|}{ AISI 1015} \\
\hline & 110 & $\mathrm{HBN}$ \\
\hline Esfuerzo a flexión & $6,63 \mathrm{ksi}$ & $6,67 \mathrm{ksi}$ \\
\hline Factor de seguridad a & 53,63 & 45,83 \\
\hline \multicolumn{3}{|c|}{ Esfuerzo a contacto } \\
\hline Esfuerzo a contacta & $12,26 \mathrm{ksi}$ & $12,36 \mathrm{ksi}$ \\
\hline \multicolumn{3}{|c|}{ Esfuerzo permisible de contacto } \\
\hline Esfuerzo permisible & $65,02 \mathrm{ksi}$ & $66,62 \mathrm{ksi}$ \\
\hline Factor de seguridad & $5,30 \mathrm{ksi}$ & 5,43 \\
\hline
\end{tabular}

Fuente: Elaboración propia

Tabla 5. Resultados del diseño de engranes cónicos (rodillos alimentadores)

\begin{tabular}{|c|c|c|c|}
\hline Torque Tp & $22,39 \mathrm{lbf}-\mathrm{in}$ & Potencia & $0,106 h p$ \\
\hline & & Piñón & Rueda \\
\hline \multicolumn{4}{|c|}{ Diseño a flexión } \\
\hline & & Piñón & Rueda \\
\hline
\end{tabular}




\begin{tabular}{|l|c|c|}
\hline Esfuerzo por Flexión & $0,797 \mathrm{ksi}$ & $1,06 \mathrm{ksi}$ \\
\hline \multicolumn{3}{|c|}{ Esfuerzo permisible Flexión } \\
\cline { 2 - 3 } & \multicolumn{2}{|c|}{ AISI 1015} \\
\hline Tipo de acero & \multicolumn{1}{|c|}{110} & HBN \\
\hline Esfuerzo a flexión & $6,63 \mathrm{ksi}$ & $6,51 \mathrm{ksi}$ \\
\hline Factor de seguridad a & 8,31 & 6,14 \\
\hline \multicolumn{3}{|c|}{ Esfuerzo a contacto } \\
\hline Esfuerzo a contacto & $23,24 \mathrm{ksi}$ & $23,24 \mathrm{ksi}$ \\
\hline \multicolumn{3}{|c|}{ Esfuerzo permisible de contacto } \\
\hline Esfuerzo permisible & $65,02 \mathrm{ksi}$ & $61,29 \mathrm{ksi}$ \\
\hline Factor de seguridad & 2,80 & 2,64 \\
\hline
\end{tabular}

Fuente: Elaboración propia

\section{Diseño de ejes de transmisión}

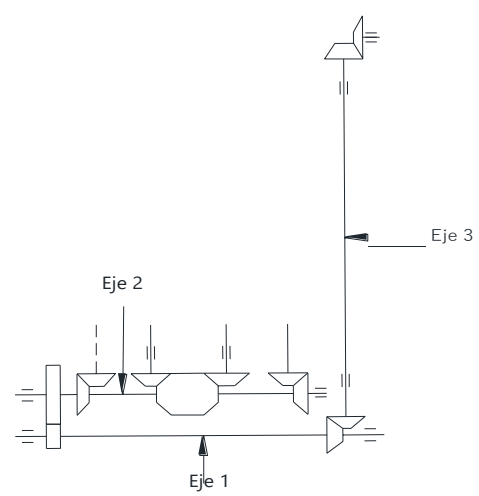

Figura 7. Esquema de ejes de transmisión para caja de engranes

La falla por fatiga es el principal causante del daño en la piezas mecánicas de las maquinas por lo que se diseñara los ejes a fatiga

Criterio lineal de soderberg

$\frac{\sigma_{a}}{S e}+\frac{\sigma_{m}}{S y}=\frac{1}{n_{f}}$

Aplicando el diseño a carga estática primero se determina el diámetro mínimo de cada eje para luego realizar un recalculo a diseño a fatiga. Los resultados se muestran en las siguientes tablas.

Tabla 6. Resultados del diseño del eje 1

\begin{tabular}{|l|c|c|}
\hline \multicolumn{3}{|c|}{ Resultados del eje y el factor de seguridad } \\
\hline Diámetro (d) & 17 & $\mathrm{~mm}$ \\
\hline \multicolumn{3}{|c|}{ Recalculo del Diseño } \\
\hline Esfuerzo Torsión: & 96,12 & $\mathrm{MPa}$ \\
\hline Esfuerzo Flexión: & 83,08 & $\mathrm{MPa}$ \\
\hline Esfuerzo Axial: & 1,57 & $\mathrm{MPa}$ \\
\hline
\end{tabular}




\begin{tabular}{|c|c|c|}
\hline Esfuerzo equivalente amplitud & 135,68 & $\mathrm{MPa}$ \\
\hline Esfuerzo equivalente medio & 242,09 & $\mathrm{MPa}$ \\
\hline Factor de seguridad & 2,07 & - \\
\hline
\end{tabular}

Fuente: Elaboración propia

Tabla 7. Resultados del diseño del eje 2

\begin{tabular}{|l|c|c|}
\hline \multicolumn{3}{|c|}{ Resultados del eje y el factor de seguridad } \\
\hline Diámetro (d) & 15 & $\mathrm{~mm}$ \\
\hline \multicolumn{3}{|c|}{ Recalculo del Diseño } \\
\hline Esfuerzo Torsión: & 5,52 & $\mathrm{MPa}$ \\
\hline Esfuerzo Flexión: & 16,64 & $\mathrm{MPa}$ \\
\hline Esfuerzo Axial: & 0,00 & $\mathrm{MPa}$ \\
\hline Esfuerzo equivalente & 61,10 & $\mathrm{MPa}$ \\
\hline Esfuerzo equivalente medio & 15,90 & $\mathrm{MPa}$ \\
\hline \multicolumn{1}{|c|}{ Factor de seguridad } & 3,21 & - \\
\hline
\end{tabular}

Fuente: Elaboración propia

Tabla 8. Resultados del diseño del eje 3

\begin{tabular}{|c|c|c|}
\hline \multicolumn{3}{|c|}{ Resultados del eje y el factor de seguridad } \\
\hline Diámetro $(\mathrm{d})$ & 13 & $\mathrm{~mm}$ \\
\hline Esfuerzo Torsión: & 48,87 & $\mathrm{MPa}$ \\
\hline Esfuerzo Flexión: & 229,22 & $\mathrm{MPa}$ \\
\hline Esfuerzo Axial: & 1,06 & $\mathrm{MPa}$ \\
\hline Esfuerzo equivalente & 317,50 & $\mathrm{MPa}$ \\
\hline Esfuerzo equivalente medio & 109,33 & $\mathrm{MPa}$ \\
\hline Factor de seguridad & 1,34 & \\
\hline
\end{tabular}

Fuente: Elaboración propia

\section{Selección del lubricante para la caja de engranes.}

La determinación del lubricante se lo realizó mediente la formula del método UNITED, que presenta Montalvo (2013), tal como se detalla a continuación.

$k=\frac{F_{t}}{b d_{p}} \cdot \frac{i+1}{i} \cdot k_{a}$

La determinación del lubricante adecuado se obtiene apartir de la viscocidad a $30^{\circ} \mathrm{C}$ a partir de la de la relación $\left(\mathrm{k} / \mathrm{V}_{\mathrm{t}}\right)$, mediante la ecuación empírica (41).

$\log \left(v_{38}\right)=-0,02767 \cdot\left[\log \left(\frac{k}{v_{t}}\right)\right]^{3}-0,10865 \cdot\left[\log \left(\frac{k}{v_{t}}\right)\right]^{2}+0,3263 \cdot \log \left(\frac{k}{v_{t}}\right)+3,0079$

Donde :

$\mathrm{V}_{\mathrm{t}}$ : velocidad tangencial $(\mathrm{m} / \mathrm{seg})$ 
Para determinar el lubricante adecuado para la caja de engranes se calculó la viscocidad cinemática en cada componente que se encontrará dentro de la caja obteniendo de esta forma que el lubricante mas adecuado sera un ISO VG 1000, o su equivalencia SAE 250.

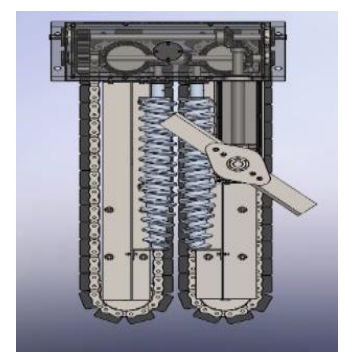

Figura 8. Modelo del mecanismo de corte

Sistema de alimentación del cosechador

\section{Tornillo sin fin alimentador de plantas}

Es el encargado de conducir las panojas hacía el acarreador para posteriormente ser trillado.

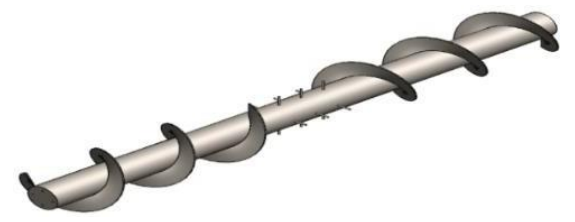

Figura 9. Modelación en 3D del tornillo sin fin

Determinación del tornillo transportador

Dónde: Velocidad angular $\omega_{\mathrm{psf}}=330 \mathrm{rpm}$, factor de llenado $\mathrm{C}_{\mathrm{f}}: 0.45$, densidad $\delta=0.49 \mathrm{gr} / \mathrm{cm}^{3}$ y $\mathrm{C}_{\mathrm{i}}=$ 1 a no estar inclinado.

$$
\begin{gathered}
D^{3}=\frac{0,0265256 * C_{p}}{\omega_{t} C_{f} \delta C_{i}} \\
D=22 c m
\end{gathered}
$$

\section{Dimensionamiento del sin fin transportador}

Diámetro del eje del transportador

$$
\mathrm{E}_{\mathrm{s}}=\mathrm{K} * \mathrm{D}
$$


$E_{s}=0,0968 m=4 i n$

Paso Pt del transportador

$$
\mathrm{P}_{\mathrm{t}}=\mathrm{F}_{\mathrm{p}} * \mathrm{D}
$$

$\mathrm{P}_{\mathrm{t}}=0,24 \mathrm{~m}$

Espesor del cuerpo del transportador

$\mathrm{Ec}=0,0104166 * \mathrm{D}$

$\mathrm{Ec}=2 \mathrm{~mm}$

\section{Determinación de la potencia por el material a transportar}

Potencia por fricción

Dónde: Longitud del transportador $\mathrm{L}=6,56 \mathrm{ft}$, velocidad angular $\mathrm{w}=330 \mathrm{rpm}$, factor de hanger $\mathrm{F}_{\mathrm{b}}=1$ y factor de diámetro del transportador $\mathrm{Fb}=31$

$\mathrm{P}_{\mathrm{f}}=\frac{\mathrm{L} * \omega * \mathrm{~F}_{\mathrm{d}} * \mathrm{~F}_{\mathrm{b}}}{1000000}$

$P_{\mathrm{f}}=0,067 \mathrm{hp}$

La potencia por el material transportado

Dónde: capacidad del transportador $\mathrm{c}=516,28 \mathrm{ft}^{3} /$ hora, densidad del material a transportar es de $30,75 \mathrm{lbs} / \mathrm{pie}^{3}$, factor de corte $\mathrm{Ff}=1$, factor de potencia de acuerdo al material $\mathrm{F}_{\mathrm{m}}=2 \mathrm{y}$ factor de mezclado $\mathrm{F}_{\mathrm{p}}=1$.

$\mathrm{P}_{\mathrm{m}}=\frac{\mathrm{c} * \mathrm{~L} * \delta * \mathrm{~F}_{\mathrm{f}} * \mathrm{~F}_{\mathrm{m}} * \mathrm{~F}_{\mathrm{p}}}{1000000}$

$\mathrm{P}_{\mathrm{m}}=0,2083 \mathrm{hp}$

Al no tener inclinación la potencia por la inclinación del transporte no se podrá calcular

Potencia total:

Dónde: Factor de sobrecarga $\mathrm{F}_{\mathrm{o}}=3$ y eficiencia e $=80 \%$. 
$P_{\text {trans }}=\frac{\left(P_{m}+P_{f}+P_{i}\right) F_{o}}{e}$

$P_{\text {trans }}=0,972 \mathrm{hp}$

Potencia debido a inerci del tornillo si fin alimentador

$\mathrm{P}_{\text {inercia }}=\frac{\left(\mathrm{T}_{\mathrm{ac}}\right) * \mathrm{n}}{7160}$

Determinación de la torsión generada por aceleración.

$$
\mathrm{T}_{\mathrm{ac}}=\mathrm{I} * \propto
$$

I: Inercia del elemento, I = 4,318kg. $\mathrm{m}^{2}$

$\alpha$ : Aceleración angular; $\alpha=2,304 \mathrm{rad} / \mathrm{seg}^{2}$

$$
\mathrm{T}_{\mathrm{ac}}=9,948 \mathrm{~N} \cdot \mathrm{m}
$$

Por lo tanto:

$P_{\text {inercia }}=0,458 \mathrm{hp}$

Potencia total para el tornillo sin fin alimentador.

$$
\begin{gathered}
\mathrm{P}_{\text {Total }}=\mathrm{P}_{\text {inercia }}+\mathrm{P}_{\text {trans }} \\
\mathrm{P}_{\text {Total }}=1,431 \mathrm{hp}
\end{gathered}
$$

Análisis de las partes constitutivas de cada sistema mediante ANSYS.

\section{Sistema de corte}

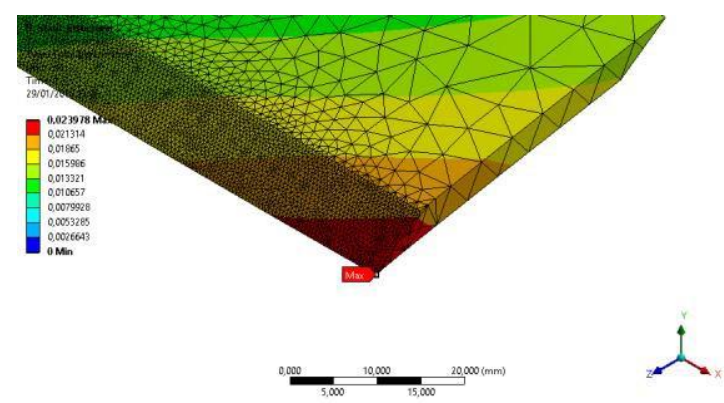

Figura 10. Deformaciones obtenidas en el análisis de la cuchilla de corte 
Tabla 9. Resultados del análisis de resistencia de la cuchilla

\begin{tabular}{|l|c|}
\hline Esfuerzo equivalente & $30,912 \mathrm{MPa}$ \\
\hline Deformación & $0,02397 \mathrm{~mm}$ \\
\hline Factor de seguridad fatiga & 8,1522 \\
\hline Convergencia & $0,16811 \%$ \\
\hline
\end{tabular}

Fuente: Elaboración propia

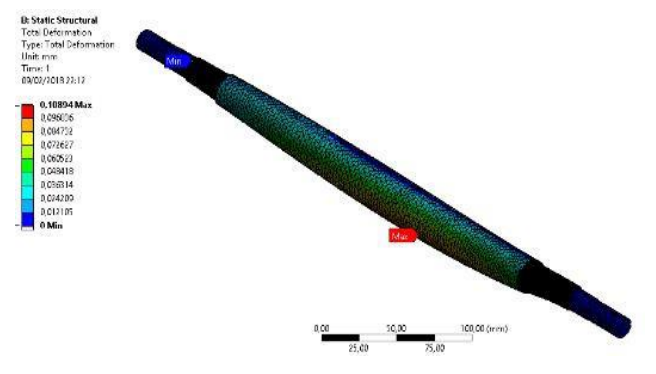

Figura 11. Análisis de ejes

Tabla 10. Resultados del análisis de resistencia de los 3 ejes.

\begin{tabular}{|c|c|c|c|c|}
\hline & Parámetros & Eje 1 & Eje 2 & Eje 3 \\
\hline $\begin{array}{c}\text { Resultados } \\
\text { Obtenidos } \\
\text { Mediante }\end{array}$ & $\sigma_{\text {equiv }}(\mathrm{MPa})$ & 267,47 & 21,28 & 119,05 \\
\cline { 2 - 5 } El & $\delta(\mathrm{mm})$ & 0,11 & 0,048 & 0,076 \\
\cline { 2 - 5 } software & $n_{f}$ & 2,04 & 7,75 & 1,75 \\
\cline { 2 - 5 } & $\begin{array}{c}\text { Convergencia } \\
\text { Cálculo } \\
\text { manual }\end{array}$ & $0,027 \%$, & $0,037 \%$ & $0,26 \%$ \\
\cline { 2 - 5 } & $\sigma_{\text {equiv }}(\mathrm{MPa})$ & 277,52 & 10,14 & 234,37 \\
\hline
\end{tabular}

Fuente: Elaboración propia 


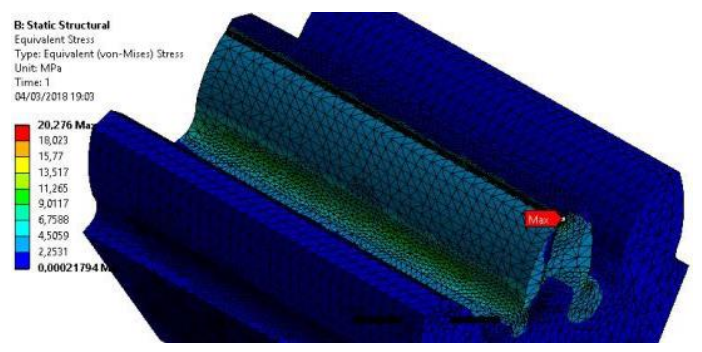

Figura 12. Deformaciones obtenidas en el Análisis de engranes

Tabla 11. Resultados del análisis de resistencia del piñón

\begin{tabular}{|l|c|}
\hline Esfuerzo equivalente & $19,23 \mathrm{MPa}$ \\
\hline Deformación & $0,000401 \mathrm{~mm}$ \\
\hline Factor de seguridad fatiga & 8,15 \\
\hline Convergencia & $2,87 \%$ \\
\hline
\end{tabular}

Fuente: Elaboración propia

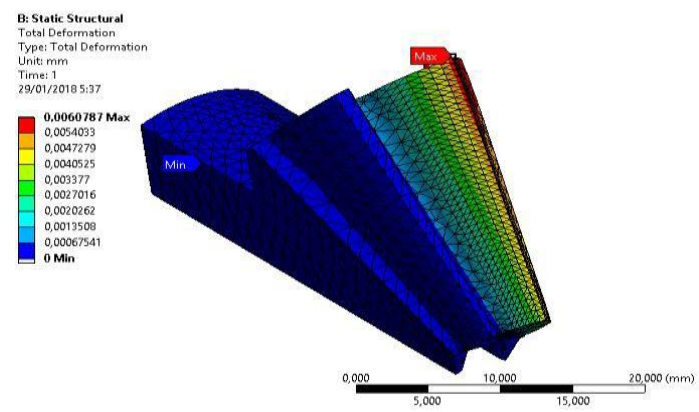

Figura 13: Deformación del diente en plena carga

Tabla 12. Resultados del análisis de resistencia de los engranes cónicos.

\begin{tabular}{|c|c|c|c|c|}
\hline Parámetros & $\begin{array}{c}\text { Engr_Con. Eje } \\
\text { corte }\end{array}$ & $\begin{array}{c}\text { Engr_Con. } \\
\text { Cuch_corte }\end{array}$ & $\begin{array}{c}\text { Engr_Con. } \\
\text { Cad_alim. }\end{array}$ & $\begin{array}{c}\text { Engr_Con. } \\
\text { Rod_alim. }\end{array}$ \\
\hline$\sigma_{\text {equiv }}(\mathrm{MPa})$ & 320,66 & 269,45 & 3,68 & 82,30 \\
\hline$\delta(\mathrm{mm})$ & 0,0061 & 0,0046 & 0,000056 & 0,00066 \\
\hline$n_{f}$ & 1,64 & 1,94 & 15 & 1,53 \\
\hline
\end{tabular}




\begin{tabular}{|c|c|c|c|c|}
\hline $\begin{array}{c}\text { Convergencia } \\
(\%)\end{array}$ & 2,33 & 3,43 & 5,12, & 2,71 \\
\hline
\end{tabular}

Fuente: Elaboración propia

Los resultados expuestos, permitieron el diseñó del sistema de corte de una máquina cosechadora integral de quinua para la región sierra del ecuador, para ello se dimensionaron las piezas que conforman el sistema de corte y se reforzó el cálculo con un análisis de resistencia en el software ANSYS, y de esta manera asegurar que todos los elementos diseñados soporten las debidas cargas de trabajo a las que estarán sometida, presentando al final un modelo del sistema de corte o cabezal, tal como se presenta en la figura 14.

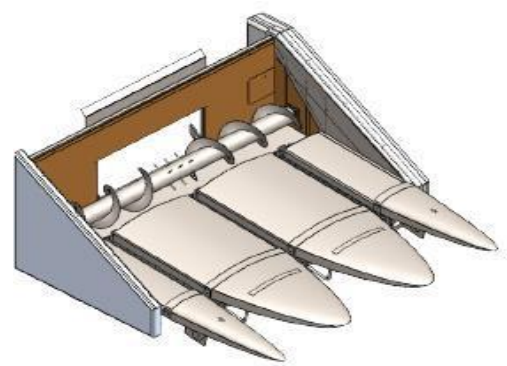

Figura 14. Modelo del sistema de corte

\section{Conclusiones}

El estudio bibliográfico permitió obtener datos de interés para el estudio, relacionado con una planta de quinua, tales como: es un producto con grandes beneficios para el ser humano y por ende de gran demanda actual, por diferentes países del mundo. También la cosecha de la quinua casi en su totalidad se realiza de forma artesanal, lo que justifica el diseño de una máquina cosechadora de quinua, para de esta forma disminuir el costo en la mano de obra, y aumentar el margen de ganancia durante su mercalización.

La información necesaria para el desarrollo del diseño del sistema de corte, se basó en los cabezales de las cosechadoras de caña de azúcar, maíz y girasol, también en datos recopilados en las entrevistas y el estudio de campo que se realizó en el canto Colta, llegando de esta manera a diseñar un sistema de corte por impacto.

Fue posible el diseñó del modelo del sistema de corte o cabezal, siendo la primera parte de la máquina cosechador integral de quinua. Todas las partes constitutivas de la máquina están 
diseñadas por medio de ecuaciones que proporcionan los libros de diseño mecánico, de mecánica agrícola y reforzando los cálculos por medio de un análisis de resistencia en software ANSYS de cada elemento crítico.

\section{Referencias Bibliográficas}

INIA (2013). Catálogo de variedades comerciales de quinua en el Perú, Quinua 2013 Año internacional, p. 23.

Cervilla, N; Mufari J y Guzman (2011). II Jornadas Investigación del NEA y países limítrofes, Argentina: Universidad Tecnología NAcional, p. 2.

Peralta, I; Mazon O; Murillo I y Rivera M. (2012). iniap.gob.ec,» 2012. [En línea]. Available: Disponible

en: http://www.iniap.gob.ec/nsite/images/documentos/MANUAL\%20AGRICOLA\%20GRANOS\%2 0ANDINOS\%202012.pdf. [Último acceso: 15 Junio 2016].

Guerrero, M. (2016). Rendimiento de quinua en el 2016-signagap,» diciembre 2016. [En línea]. Available:

http://sinagap.agricultura.gob.ec/pdf/estudios_agroeconomicos/rendimiento_quinua.pdf. [Último acceso: 02 junio 2017].

Norton (2009). Diseño de maquinaria, México, D.F.: McGrawHill.

Hamrock, J; Jacbson y Schmid, S. (2000). Elementos de máquinas, México, D.F.: McGraw-Hill.

Ortiz, J., (2012). Valores de rendimiento efectivo o de campo para diferentes labores,» de Las máquinas agrívolas y su aplicación, Madrid-España, Mundi Prensa, pp. 529,531.

Montalvo, D.(2013). INGEMECANICA. [En línea]. Available: Disponible en: https://ingemecanica.com/tutorialsemanal/objetos/tutorial159.pdf. [Último acceso: 13 Mayo 2017]. 